\title{
Brain maturation is delayed in infants with complex congenital heart defects
}

\author{
Daniel J. Licht, MD, ${ }^{\mathrm{a}}$ David M. Shera, ScD, ${ }^{\mathrm{b}}$ Robert R. Clancy, MD, ${ }^{\mathrm{a}}$ Gil Wernovsky, MD,${ }^{\mathrm{c}}$ Lisa M. Montenegro, MD, ${ }^{\mathrm{d}}$ \\ Susan C. Nicolson, MD, ${ }^{\mathrm{d}}$ Robert A. Zimmerman, MD,${ }^{\mathrm{e}}$ Thomas L. Spray, MD, ${ }^{\mathrm{f}}$ J. William Gaynor, MD, ${ }^{\mathrm{f}}$ and \\ Arastoo Vossough, $\mathrm{MD}^{\mathrm{e}}$
}

Objective: Small head circumferences and white matter injury in the form of periventricular leukomalacia have been observed in populations of infants with severe forms of congenital heart defects. This study tests the hypothesis that congenital heart defects delay in utero structural brain development.

\begin{abstract}
Methods: Full-term infants with hypoplastic left heart syndrome or transposition of the great arteries were prospectively evaluated with preoperative brain magnetic resonance imaging. Patients with independent risk factors for abnormal brain development (shock, end-organ injury, or intrauterine growth retardation) were excluded. Outcome measures included head circumferences and the total maturation score on magnetic resonance imaging. Total maturation score is a previously validated semiquantitative anatomic scoring system used to assess whole brain maturity. The total maturation score evaluates 4 parameters of maturity: (1) myelination, (2) cortical infolding, (3) involution of glial cell migration bands, and (4) presence of germinal matrix tissue.
\end{abstract}

Results: The study cohort included 29 neonates with hypoplastic left heart syndrome and 13 neonates with transposition of the great arteries at a mean gestational age of $38.9 \pm 1.1$ weeks. Mean head circumference was 1 standard deviation below normal. The mean total maturation score for the cohort was $10.15 \pm 0.94$, significantly lower than reported normative data in infants without congenital heart defects, corresponding to a delay of 1 month in structural brain development.

Conclusion: Before surgery, term infants with hypoplastic left heart syndrome and transposition of the great arteries have brains that are smaller and structurally less mature than expected. This delay in brain development may foster susceptibility to periventricular leukomalacia in the preoperative, intraoperative, and postoperative periods.

\begin{abstract}
Advances in the medical and surgical care of children with severe forms of congenital heart defects (CHDs) have led to increasing numbers of children surviving and attending school. There is a growing recognition of neurocognitive impairments in this population. Previous investigations have sought a better understanding of the basis of these deficits and have primarily focused on the modification of intraoperative variables, such as the duration of cardiopulmonary bypass, the use and duration of deep hypothermic circulatory arrest, the hematocrit level, and the management of $\mathrm{pH}$ status. ${ }^{1-3}$ Other studies have found that patient-specific factors, such as the cardiac diagnosis, genetic syndromes, and polymorphisms of the gene for apolipoprotein $\mathrm{E}(A P O E),{ }^{4,5}$ were
\end{abstract}

\footnotetext{
From the Divison of Neurology, ${ }^{\mathrm{a}}$ Biostatistics and Epidemiology, ${ }^{\mathrm{b}}$ Cardiology and Critical Care Medicine, ${ }^{\mathrm{c}}$ Division of Cardiac Anesthesia, ${ }^{\mathrm{d}}$ Division of Neuroradio$\operatorname{logy},{ }^{\mathrm{e}}$ and Division of Cardiothoracic Surgery, ${ }^{\mathrm{f}}$ The Children's Hospital of Philadelphia, Philadelphia, Pennsylvania.

Dr Licht is supported by Grant K23-NS052380.

Received for publication May 8, 2008; revisions received Sept 23, 2008; accepted for publication Oct 26, 2008.

Address for reprints: Daniel J. Licht, MD, Division of Neurology, The Children's Hospital of Philadelphia, 3535 Market St, Room 1433, Philadelphia, PA 19104 (E-mail: licht@email.chop.edu).

J Thorac Cardiovasc Surg 2009;137:529-37

$0022-5223 / \$ 36.00$

Copyright (c) 2009 by The American Association for Thoracic Surgery

doi:10.1016/j.jtcvs.2008.10.025
}

additional and potentially stronger predictors of long-term neurocognitive outcomes than intraoperative variables.

In the neonatal period, abnormalities of the central nervous system are common in patients with complex CHDs. These include clinical findings, such as hypotonia, seizures, feeding difficulties, and abnormalities seen on brain imaging, such as stroke, hemorrhage, and periventricular leukomalacia (PVL) ${ }^{6-9}$ In preterm neonates without CHD, PVL is the most commonly identified neuropathologic lesion with its frequency and severity proportional to the degree of prematurity. The high occurrence of PVL in premature neonates is attributed to the enhanced vulnerability of premyelinating oligodendrocyte precursor cells to hypoxia/ ischemia, which are present in their highest concentrations from 23 to 32 weeks of gestation but persist in lower concentrations until 34 to 36 weeks. ${ }^{10}$ Identification of PVL on preoperative magnetic resonance imaging (MRI) scans of infants with CHD suggests increased vulnerability of white matter in these children, raising concerns about brain and white matter maturity. The question of brain immaturity has been raised ${ }^{11}$ and recently supported by evidence of biochemical immaturity of the white matter as determined by preoperative MRI spectroscopy and diffusion tensor imaging. ${ }^{12}$

This study tests the hypothesis that brain development is structurally delayed in infants with complex CHD using 

Abbreviations and Acronyms
CHD = congenital heart defect
$\mathrm{ECA}=$ extracardiac anomaly
EGA $=$ estimated gestational age
$\mathrm{GA}=$ gestational age
HLHS $=$ hypoplastic left heart syndrome
MRI = magnetic resonance imaging
PVL = periventricular leukomalacia
TGA $=$ transposition of the great arteries
TMS $=$ total maturation score

the total maturation score (TMS), a semiquantitative scoring system developed and validated in healthy preterm infants. ${ }^{13}$ The TMS system has also been used to test the effect of PVL on whole brain maturation in preterm infants imaged at term. ${ }^{14}$

\section{MATERIALS AND METHODS Patients}

The study was approved by the institutional review board at The Children's Hospital of Philadelphia. Written consent was obtained from the parents/guardians of all patients enrolled. Inclusion criteria were "healthy" term infants (gestational age [GA] > 37 weeks) with either hypoplastic left heart syndrome (HLHS) or transposition of the great arteries (TGA). Exclusion criteria included factors that would independently affect brain maturity, such as intrauterine growth retardation or perinatal factors that could independently affect MRI findings, such as a history of perinatal depression (APGAR score $<5$ at 5 minutes or $\mathrm{pH}<7.0$ ), evidence of end-organ injury (liver function tests $>2 \times$ upper limit normal, creatinine $>2 \mathrm{mg} / \mathrm{dL}$, encephalopathy, or seizures), or evidence of intracranial hemorrhage on head ultrasound. Head circumferences were measured by a single child neurologist (D.J.L.). Extracardiac anomalies (ECAs), such as low-set ears or micrognathia, were noted on preoperative physical examination and considered potential markers for underlying genetic disorders.

\section{Study Protocol}

All patients were prepared for surgery following our standard clinical protocols. ${ }^{8}$ On the morning of surgery, patients were brought to the operating room by the cardiac anesthesia team to induce anesthesia (fentanyl 5-10 $\mu \mathrm{g} / \mathrm{kg}$, pancuronium $0.2 \mathrm{mg} / \mathrm{kg}$ ), perform endotracheal intubation, and secure vascular access. Patients were then transported to the MRI suite. Heart rate, blood pressure, electrocardiogram, transcutaneous oxygen saturations, and end-tidal $\mathrm{CO}_{2}$ measurements were monitored throughout transport and during the performance of the MRI.

\section{Structural Magnetic Resonance Imaging}

All MRIs were acquired on a Siemens 3.0T Trio (New York, NY) at the Children's Hospital of Philadelphia. Studies included volumetric T1 and T2 sampling perfection with application-optimized contrasts using different flip angle evolution sequences acquired in the axial plane and later reconstructed in the sagittal and coronal planes. Axial fluid attenuated inversion recovery sequence, susceptibility sequences (both standard echo gradient and susceptibility weighted imaging), and diffusion weighted sequences were also acquired.

\section{Image Interpretation}

Clinical MRI interpretations were performed by a single pediatric neuroradiologist (R.A.Z.) blinded to the patient's clinical information. Imaging abnormalities were identified and localized by their signal intensity, appearance, and acuity, as judged by the presence or absence of restriction of water movement on diffusion weighted sequences. Apparent diffusion coefficient maps were correlated to diffusion positive lesions to confirm ischemic changes. Hemorrhage, ischemia, and structural abnormalities of the brain or blood vessels were catalogued. PVL was recognized primarily as T1 hyperintensity and corresponding T2 signal loss in the periventricular white matter. These lesions were graded as mild: one to several punctate areas of increased T1 signal $(<3 \mathrm{~mm})$; moderate: multiple small areas $(3 \mathrm{~mm}-1$ $\mathrm{cm})$ that were not confluent; or severe: large, confluent areas greater than $1 \mathrm{~cm}$. The finding of an open operculum was based on visual inspection of the frontal, temporal, and parietal lobes and their relations around the Sylvian fissure overlying the insular cortex. An underdeveloped, or "open," operculum was diagnosed on the basis of prior knowledge of normal interopercular distances. ${ }^{15}$

\section{Total Maturation Score}

The TMS system described by Childs ${ }^{13}$ was used (Table 1). TMS evaluations were carried out by 2 independent observers (D.J.L., A.V.) blinded to clinical data. Axial T1 and T2 images were used for the evaluations; special attention was focused on slices near the foramen of Monro (Figure 1). Myelination was scored separately on $\mathrm{T} 1$ and $\mathrm{T} 2$ for each hemisphere. Scores were then averaged to yield the final myelination subscore. Conversely, the cortical folding score was composed of the average of individual scoring items identified in the original article ${ }^{13}$ (Table 1).

\section{Statistical Methods}

Simple descriptive statistics were used to characterize the demographics of the population. Pearson's correlation and intraclass correlation statistics were calculated to define the interrater reliability for total TMS score and subscales, and a Bland-Altman plot was constructed. Correlations of brain maturity scores with head circumference, brain volumes, and risk for PVL were assessed with $t$ statistics, stepwise logistic analyses, and linear regression analyses.

\section{RESULTS}

Between February 2006 and December 2007, 60 families were approached for consent and 44 families gave consent for participation. Two patients initially enrolled were not included secondary to medical instability in the 12 hours before surgery. The remaining 42 patients $(29[69 \%, 20$ with aortic atresia] with HLHS and 13 [31\%, 12 with intact ventricular septum] with TGA) underwent brain MRI at $4.1 \pm$ 2.4 days of life (mean \pm standard deviation, range 1-13 days). A prenatal diagnosis had been made in 29 patients $(24 / 29$ [83\%] with HLHS and 5/13 [39\%] with TGA, $P<$ .01 ). The GA of those patients with a prenatal diagnosis of their heart defect was not different from that of patients with a postnatal diagnosis $(38.8 \pm 1.2$ weeks and $39.1 \pm$ 1.1 weeks, respectively, $P=.464$ ). Table 2 summarizes the patient characteristics. The predominant gender was male $(27 / 42,64 \%)$, and the mean GA was $38.9 \pm 1.1$ weeks (range 36.5-41 weeks). Mean birth head circumference at birth was $34.6 \pm 1.1 \mathrm{~cm}$, and birth weight was $3.37 \pm$ $0.53 \mathrm{~kg}$. The mean head circumference was 1 standard deviation below the expected value for age. Patients with HLHS 
TABLE 1. Total maturation score system

\begin{tabular}{|c|c|}
\hline \multicolumn{2}{|c|}{ Myelination score } \\
\hline M1 & Myelination evident in the brainstem, cerebellar peduncle, inferior colliculus, cerebellar vermis \\
\hline M2 & M1 + Subthalamic nucleus, globus pallidus, ventrolateral thalamus \\
\hline M3 & M2 + Caudal portion of the PLIC \\
\hline M4 & $\mathrm{M} 3$ + Complete PLIC \\
\hline M5 & M4 + Corona radiata \\
\hline M6 & M5 + Anterior limb of the internal capsule \\
\hline \multicolumn{2}{|c|}{$\begin{array}{l}\text { Cortical folding, opercular folding, and T1 } \\
\text { intensity score }\end{array}$} \\
\hline \multicolumn{2}{|c|}{ Cortical folding ${ }^{\mathrm{a}}$} \\
\hline $\mathrm{C} 1$ & Frontal and occipital cortex is completely smooth \\
\hline $\mathrm{C} 2$ & Frontal cortex is smooth, some sulci evident in occipital cortex \\
\hline $\mathrm{C} 3$ & Frontal and occipital cortex demonstrating similar number of sulci \\
\hline $\mathrm{C} 4$ & Frontal and occipital cortex "rich" in sulci, sulcation along the frontal interhemispheric fissure \\
\hline C5 & Frontal and occipital WM separated into strands by deeper sulci \\
\hline C6 & $\mathrm{C} 5+$ isointense $\mathrm{WM}$ on $\mathrm{T} 1$ \\
\hline \multicolumn{2}{|c|}{ Insular cortex ${ }^{\mathrm{a}}$} \\
\hline $\mathrm{C} 1$ & Insula wide open with thin, bright cortical rim \\
\hline $\mathrm{C} 2$ & Insula still wide open with almost smooth surface \\
\hline $\mathrm{C} 3$ & Internal surface of the insula more convoluted \\
\hline $\mathrm{C} 4$ & Progression of cortical infolding on the insular surface \\
\hline C5 & Insula completely infolded \\
\hline \multicolumn{2}{|c|}{ T1 WM intensity ${ }^{\mathrm{a}}$} \\
\hline $\mathrm{C} 1$ & WM "entirely" low intensity on T1 \\
\hline $\mathrm{C} 2$ & WM "entirely" low intensity on T1 \\
\hline $\mathrm{C} 3$ & WM “somewhat" low intensity \\
\hline $\mathrm{C} 4$ & WM "slightly" low intensity \\
\hline C5 & WM indistinguishable from GM \\
\hline C6 & WM indistinguishable from GM \\
\hline \multicolumn{2}{|c|}{ Germinal matrix } \\
\hline G1 & Matrix seen in posterior horn, CTN, and anterior horns of the lateral ventricles \\
\hline G2 & Matrix seen at CTN and anterior horns of the lateral ventricles \\
\hline G3 & Matrix seen in the anterior horns only \\
\hline G4 & No germinal matrix evident \\
\hline \multicolumn{2}{|c|}{ Bands of migrating glial cells } \\
\hline B1 & Broad band with additional narrower bands evident \\
\hline B2 & Broad band alone \\
\hline B3 & Narrow band alone \\
\hline B4 & No bands seen \\
\hline
\end{tabular}

CTN, Caudothalamic notch; PLIC, posterior limb of the internal capsule; $W M$, white matter; GM, gray matter. ${ }^{\text {a }}$ Scores were applied to both hemispheres and then averaged for the whole brain score.

were slightly younger than patients with TGA $(38.8 \pm 1.2 \mathrm{vs}$ $39.3 \pm 0.9$ weeks, respectively, $t$ test $P=.08)$.

ECAs occurred in 12 of 42 patients $(29 \%, 3 / 13$ with TGA and 9/29 with HLHS, Fisher's exact test $P=.7225$ ). Examples included low-set, posteriorly rotated ears, micrognathia, webbed neck, and hypospadias. Seven patients had multiple anomalies. Patients with ECAs did not differ significantly from patients without ECAs in TMS scores, head circumference, or birth weight (Table 3 ).

\section{Structural Magnetic Resonance Imaging}

Clinical MRI interpretation revealed PVL in 9 of 42 patients ( $21 \%, 4 / 29$ with HLHS, 5/13 with TGA, Fisher's exact $P=$ $.107)$ and stroke in 4 of 42 patients $(9.5 \%, 4 / 29$ with HLHS, $0 / 13$ with TGA, Fisher's exact $P=.293$ ). PVL was moderate in 6 of 9 patients $(67 \%)$ and mild in the others. Incomplete closure of the opercular space, a radiologic sign of brain immaturity, was seen in 36 of 42 patients $(86 \%, 24 / 29$ with HLHS, $12 / 13$ with TGA, $P=.65$ ). Stepwise logistic regression analysis failed to identify significant associations for increased risk for PVL as a dichotomous variable (present/absent). Multivariate stepwise analysis using PVL as a categoric variable (none/ mild/moderate/severe) showed increasing risk with decreasing GA $(P=.014)$. Of the 12 patients with an estimated GA (EGA) of 40 weeks, only 1 had MRI evidence of PVL (mild).

\section{Total Maturation Score}

Interclass correlation showed good agreement (interclass correlation coefficient $=0.712$ ) between raters. Bland-Altman 

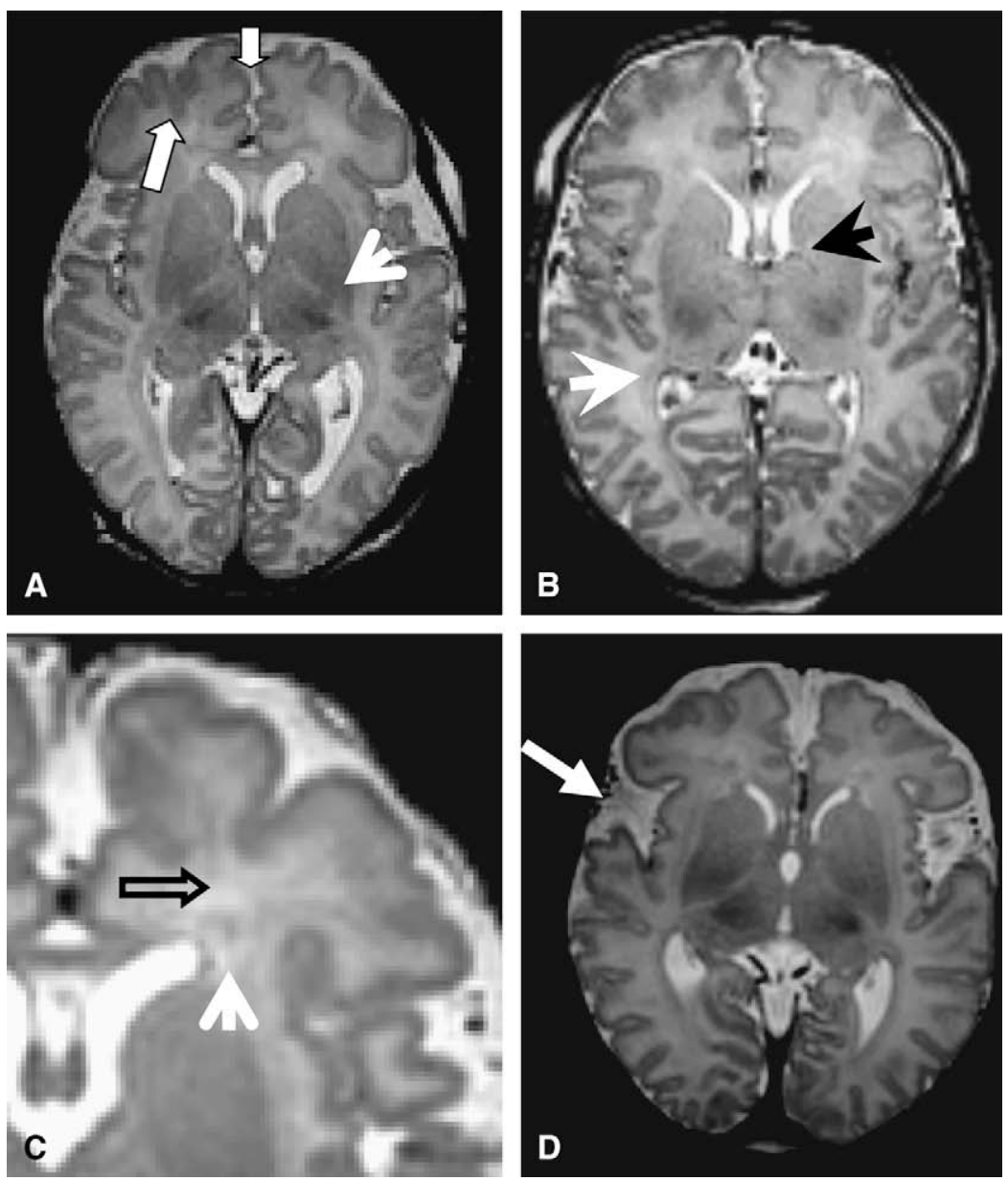

FIGURE 1. Scoring samples. A, Block arrows point to deep frontal and interhemispheric sulcation (score $=\mathrm{C} 4)$; arrowhead demonstrates posterior limb of internal capsule with myelination (score $=\mathrm{M} 3$ ). B, Arrows point to germinal matrix still present in posterior (white arrow) and anterior (black arrow) lateral ventricle (score = GM1). C, Arrows demonstrate 2 bands of migrating glial cells (score B1). D, Bilaterally underdeveloped opercular spaces (white arrow).

Plot (Figure 2) showed a mean difference between raters of $0.39 \pm 0.7$. The TMS increased linearly with EGA ( $r=$ 0.42 ), and the average TMS for the entire cohort was $10.15 \pm 0.94$ (range 8.3-12.3). This is significantly lower than the mean TMS scores reported for infants without $\mathrm{CHD}^{13}$ (Figure 3) of comparable GA (36-37 weeks $\mathrm{n}=$ 28 , mean TMS $=11.1 \pm 1.5, P=.0045,38-43$ weeks, $\mathrm{n}=16$, mean TMS $=13.0 \pm 2.3, P<.0001)$. Study TMS scores were also significantly lower than the TMS scores of premature infants born who were then imaged at term (TMS score of $14.00 \pm 1.4$ for preterm infants without PVL and $12.44 \pm 2.3$ with PVL). ${ }^{14}$ The mean TMS of the study group compared with the equivalent GA of 35.1 weeks in children without CHD, suggesting that the study group with CHD had brain maturation of approximately 1 month younger than their actual GA.

TABLE 2. Patient characteristics

\begin{tabular}{|c|c|c|c|c|}
\hline & Total $n=42$ & TGA $n=13$ & HLHS n $=29$ & $P$ Value \\
\hline Day of life & $4.1 \pm 2.4$ & $4.5 \pm 3.0$ & $3.9 \pm 2.0$ & .56 \\
\hline Gestational age & $38.9 \pm 1.1$ & $39.3 \pm 0.9$ & $38.7 \pm 1.2$ & .08 \\
\hline Birth weight & $3.4 \pm 0.5$ & $3.5 \pm 0.5$ & $3.3 \pm 0.5$ & .18 \\
\hline Head circumference & $34.6 \pm 1.1$ & $34.8 \pm 1.1$ & $34.5 \pm 1.1$ & .44 \\
\hline PVL & $9(21 \%)$ & $5(39 \%)$ & $4(14 \%)$ & .11 \\
\hline Stroke & $4(9.5 \%)$ & $0(0 \%)$ & $4(14 \%)$ & .29 \\
\hline TMS score & $10.15 \pm 0.94$ & $10.27 \pm 1.0$ & $10.10 \pm 0.9$ & .61 \\
\hline
\end{tabular}

TGA, Transposition of the great arteries; HLHS, hypoplastic left heart syndrome; $P V L$, periventricular leukomalacia; $T M S$, total maturation score. 
TABLE 3. Effect of extracardiac anomalies on patient characteristics

\begin{tabular}{lccc}
\hline & ECA $\mathbf{n}=\mathbf{1 2}$ & No ECA $\mathbf{n}=\mathbf{3 0}$ & $\boldsymbol{P}$ Value \\
\hline TGA & $3(25 \%)$ & $10(33 \%)$ & .73 \\
HLHS & $9(75 \%)$ & $20(67 \%)$ & \\
Day of life & $4.67 \pm 3.6$ & $3.9 \pm 1.7$ & .47 \\
Gestational age & $38.5 \pm 1.3$ & $39.0 \pm 1.0$ & .27 \\
Birth weight & $3.4 \pm 0.5$ & $3.4 \pm 0.5$ & .91 \\
Head circumference & $34.3 \pm 1.1$ & $34.7 \pm 1.1$ & .24 \\
TMS score & $10.01 \pm 0.96$ & $10.21 \pm 0.96$ & .53 \\
PVL & $3(25 \%)$ & $6(25 \%)$ & .70 \\
\hline
\end{tabular}

$E C A$, Extracardiac anomaly; $T G A$, transposition of the great arteries; $T M S$, total maturation score; $P V L$, periventricular leukomalacia.

In this study, stepwise analysis of study TMS scores revealed a positive correlation with $\mathrm{GA}(P=.006)$. However, TMS scores were not different between infants with HLHS and infants with TGA or between infants with and without ECAs (Tables 2 and 3).

\section{DISCUSSION}

Neurodevelopmental delays constitute the most common morbidity for school-aged children with complex CHD who required cardiac surgery as neonates or young infants, ${ }^{1,16}$ because approximately half require remedial academic services. ${ }^{17,18}$ Studies from multiple centers of children with various types of CHD show a surprisingly similar pattern, including relatively preserved intelligence but a higher than expected frequency of deficits in attention, executive function, language, fine and gross motor coordination, and visual-motor integration. Their neurodevelopmental profile is qualitatively similar to that of premature infants. Both groups share a common neuropathology, PVL, which is an injury to the white matter located in a vascular watershed zone adjacent to the lateral ventricles. Hypoxia-ischemia is a common cause of PVL, and the vulnerability of the white matter to hypoxia-ischemia is heavily dependent on brain maturity and the concentration of vulnerable cell populations, known as late oligodendrocyte progenitors. ${ }^{10}$ The density of these delicate premyelinating oligodendrocyte progenitor cells peaks at a GA of 23 to 32 weeks but may persist until 35 to 36 weeks. This corresponds to a period of peak PVL prevalence in preterm infants. The high prevalence of PVL among term infants with CHD in the preoperative, intraoperative, and postoperative periods suggests that they also demonstrate an enhanced susceptibility to white matter injury from brain immaturity, possibly related to the CHD.

Well-designed investigations of neurodevelopmental outcomes of infant heart surgery have focused on intraoperative variables such as bypass strategies, hematocrit, and $\mathrm{pH}$ management but have failed to show clear and consistent long-term benefit. ${ }^{1,2,19-21}$ Indeed, the Bayley scales of infant development in infants after TGA repair have not changed substantially over the past 2 decades, despite many modifications in intraoperative strategy. ${ }^{1,19,21,22}$ Although intraoperative techniques are undoubtedly important, the lack of significant improvement in developmental scores in TGA suggests that it is the underlying brain substrate in CHD in general, and TGA in particular, that contributes more significantly to longer-term outcomes than changes in intraoperative support techniques. There is a growing recognition of the stronger contribution of patient factors, such as cardiac diagnosis, genetic diagnoses, and $A P O E$ genotype, as important determinants of neurodevelopmental outcomes. ${ }^{4,5}$ Furthermore, there is greater appreciation that in $\mathrm{CHD}$, the brain is smaller than expected and commonly shows white matter injury, both before and after surgery, suggesting immaturity. ${ }^{6-8,11}$

\section{Total TMS}

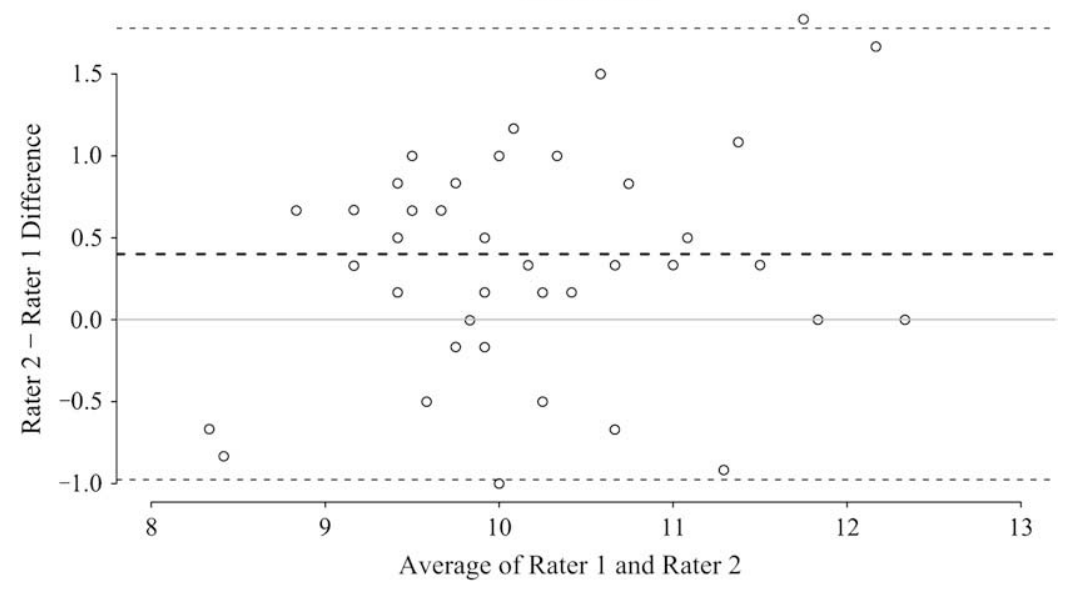

FIGURE 2. Interrater reliability. Bland-Altman plot demonstrating interrater differences. The mean difference was $-0.35 \pm 0.7$ (dotted lines, bold $=$ mean, light $=2$ standard deviations from mean). TMS, Total maturation score. 


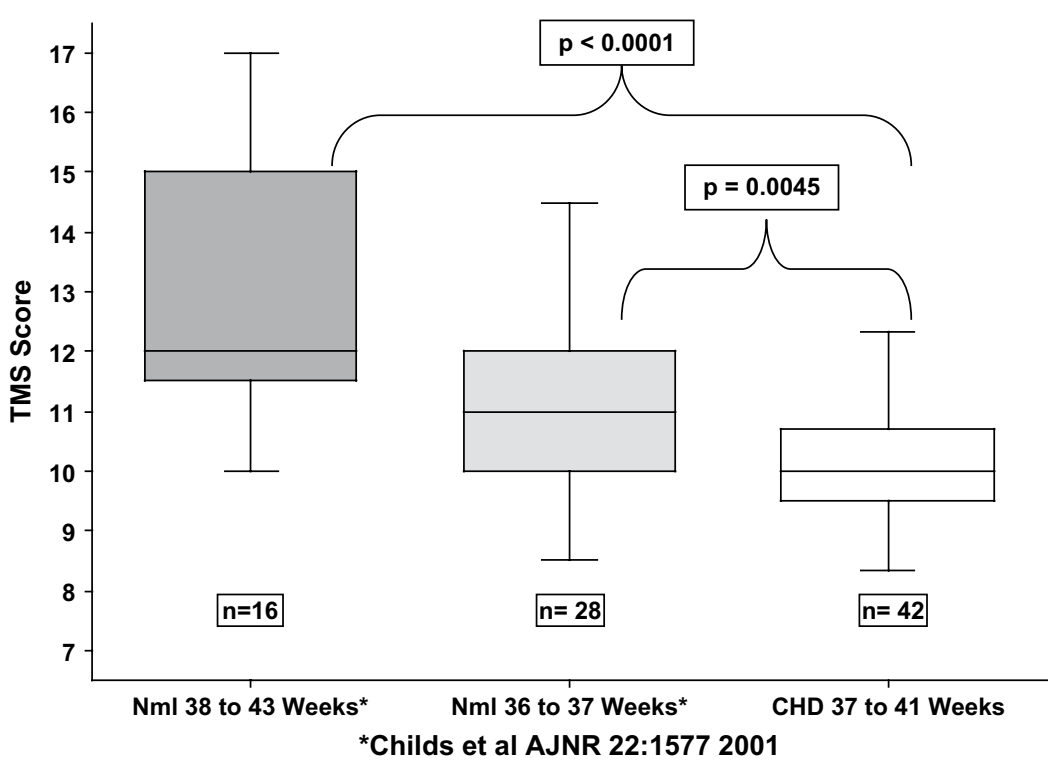

FIGURE 3. TMS. Box and whisker plots comparing the TMS of infants with CHD (open box, far right) to typically developing infants represented in the solid boxes (dark gray 38-43 weeks GA, light gray 36-37 weeks GA). TMS, Total maturation score; CHD, congenital heart defect.

This study tests the hypothesis that brain development is structurally delayed in infants with complex CHD. Brain development involves a rapid and predictable sequence of structural alterations of cortical and subcortical structures. In very early fetal life, the cortex begins as a smooth structure, followed by the onset of sulcation at 20 weeks EGA with the gradual appearance of deep primary and more superficial secondary cortical infoldings. The germinal matrices, sites of origin, and migration of cortical neurons and glial cells appear robust between 10 and 28 weeks EGA. The blood supply to the germinal matrix then gradually involutes as neuronal proliferation halts and glial multiplication (forming migrating bands of glial cells) begins, starting at 20 weeks EGA. Glial cell migration then continues postnatally. ${ }^{23}$ The myelination of the cerebral hemispheres is largely postnatal but can be detected in the optic radiations and posterior limbs of the internal capsule beginning at approximately 35 weeks of gestation and progresses steadily thereafter through age 2 years. ${ }^{23}$ These predictable anatomic maturational sequences can be measured by the TMS, a semiquantitative scoring system developed and validated in healthy preterm infants. ${ }^{13}$

TMS scores in our cohort were significantly lower than those of normal controls of similar GA ( $>36$ weeks GA). The average TMS score for the study group with TGA and HLHS was at the 50th percentile for 35 weeks gestation, 4 weeks below their actual mean GA. These observations of anatomic immaturity complement a recent study of biochemical "immaturity" measured by magnetic resonance spectroscopy and diffusion tensor imaging. ${ }^{12}$ Consistent with the TMS scores, their mean head circumference was smaller than expected for their gestation age: a full standard deviation below normal. This agrees with a previous study from our group using novel semiautomated MRI techniques that demonstrated reduced brain volumes for age. ${ }^{24}$ Further, immature brain development was also seen in the incomplete closure of the cerebral operculae in approximately $90 \%$ of the infants studied. The operculum comprises an area of brain that includes the sensory motor cortical representation of buccal, glottic, and esophageal structures, as well as receptive and expressive language. Bilateral abnormalities in the cerebral operculum have been linked to feeding and language delays. ${ }^{15}$

Our cohort of preoperative infants with CHD was specifically selected to exclude patients with intrauterine growth retardation, as well as those at increased risk for brain injury from perinatal distress. Despite this selection of "lowerrisk" infants, PVL was identified in more than $20 \%$ of neonates before surgery, similar to that seen in previous studies. ${ }^{6-9}$ Consistent with the hypothesis that the central nervous system in these infants is immature and thus "vulnerable" to hypoxia and ischemia, the incidence of PVL increases to at least $50 \%$ after surgery. ${ }^{9,25}$ Factors related to the increase or severity of PVL have included adverse postoperative hemodynamics, including hypoxemia, hypotension, ${ }^{25}$ and low systemic oxygen saturation as measured by near infrared spectroscopy, ${ }^{9}$ rather than factors related to intraoperative management strategies.

Low TMS scores, small head circumferences, a high prevalence of open operculae, and an increased and ongoing risk for PVL collectively suggest that in utero brain development is impaired in this population. In the fetus without CHD, the normal fetal circulation results in preferential streaming of blood with higher oxygen content from the placenta to the 
left atrium and ventricle, and then to the brain. This pattern is not present in TGA, where the aorta is aligned with the right ventricle, or in HLHS, where the left ventricle and aorta are hypoplastic or virtually absent. These altered fetal blood flow patterns are associated with measurable changes in fetal cerebral vascular resistance, ${ }^{26,27}$ as well as the potential for reduced oxygen delivery, which likely lead to abnormalities of brain development. Consistent with this hypothesis, we have previously noted that reduced head circumference in infants with HLHS was significantly associated with smaller diameters of the ascending aorta ${ }^{28}$ and microcephaly, as previously noted in many studies of $\mathrm{CHD}^{4,5,8}$ In vivo experiments have demonstrated that oligodendrocyte maturation can be delayed in rat pups raised in a hypoxic environment and predisposes the brain to PVL at an age that it would not be otherwise expected. ${ }^{29}$ Similar mechanisms may be at play with severe forms of CHD.

\section{Limitations}

The current study is limited by its relatively modest size. Nonetheless, the frequency of microcephaly, PVL, and open opercula is consistent with previous reports and our larger cumulative experience with preoperative brain MRI in 102 infants with complex CHD. It also must be emphasized that this study was limited to neonates with 2 cardiac diagnoses-HLHS and TGA - and the incidence of congenital brain abnormalities may be different in other populations with CHD.

\section{CONCLUSIONS}

The findings in the current study suggest that the brains of term neonates with TGA or HLHS appear to be delayed by approximately 1 month. Although the causes are almost certainly multifactorial, it is likely that altered fetal blood flow patterns and diminished oxygen delivery play an important role. These findings have important implications for obstetric management of neonates with a CHD diagnosis in the prenatal period, because a prenatal diagnosis may prompt induction of labor before term. ${ }^{30}$ In our cohort, TMS scores increased with increasing GA, suggesting that waiting the full 40 weeks may be beneficial. Our findings also emphasize the importance of preoperative brain imaging in future neuroprotection studies to allow proper risk stratification in various treatment arms.

\section{References}

1. Bellinger DC, Wypij D, duDuplessis AJ, Rappaport LA, Jonas RA, Wernovsky G, et al. Neurodevelopmental status at eight years in children with dextro-transposition of the great arteries: the Boston Circulatory Arrest Trial. J Thorac Cardiovasc Surg. 2003;126:1385-96.

2. Bellinger DC, Wypij D, du Plessis AJ, Rappaport LA, Riviello J, Jonas RA, et al. Developmental and neurologic effects of alpha-stat versus $\mathrm{pH}$-stat strategies for deep hypothermic cardiopulmonary bypass in infants. J Thorac Cardiovasc Surg. 2001;121:374-83.

3. de Ferranti S, Gauvreau K, Hickey PR, Jonas RA, Wypij D, du Plessis A, et al. Intraoperative hyperglycemia during infant cardiac surgery is not associated with adverse neurodevelopmental outcomes at 1, 4, and 8 years. Anesthesiology. 2004;100:1345-52

4. Gaynor JW, Gerdes M, Zackai EH, Bernbaum J, Wernovsky G, Clancy RR, et al. Apolipoprotein E genotype and neurodevelopmental sequelae of infant cardiac surgery. J Thorac Cardiovasc Surg. 2003;126:1736-45.

5. Gaynor JW, Wernovsky G, Jarvik GP, Bernbaum J, Gerdes M, Zackai E, et al. Patient characteristics are important determinants of neurodevelopmental outcome at one year of age after neonatal and infant cardiac surgery. J Thorac Cardiovasc Surg. 2007;133:1344-53, 53 e1-3.

6. Mahle WT, Tavani F, Zimmerman RA, Nicolson SC, Galli KK, Gaynor JW, et al. An MRI study of neurological injury before and after congenital heart surgery. Circulation. 2002;106:I109-14.

7. McQuillen PS, Barkovich AJ, Hamrick SE, Perez M, Ward P, Glidden DV, et al. Temporal and anatomic risk profile of brain injury with neonatal repair of congenital heart defects. Stroke. 2007;38:736-41.

8. Licht DJ, Wang J, Silvestre DW, Nicolson SC, Montenegro LM, Wernovsky G, et al. Preoperative cerebral blood flow is diminished in neonates with severe congenital heart defects. J Thorac Cardiovasc Surg. 2004;128:841-9.

9. Dent CL, Spaeth JP, Jones BV, Schwartz SM, Glauser TA, Hallinan B, et al. Brain magnetic resonance imaging abnormalities after the Norwood procedure using regional cerebral perfusion. J Thorac Cardiovasc Surg. 2005;130:1523-30.

10. Back SA, Luo NL, Borenstein NS, Levine JM, Volpe JJ, Kinney HC. Late oligodendrocyte progenitors coincide with the developmental window of vulnerability for human perinatal white matter injury. $J$ Neurosci. 2001;21:1302-12.

11. Licht DJ, Agner S, Montenegro LM, Nicolson SC, Silvestre D, Tabbutt S, et al. Preoperative MRI abnormalities are common in full-term infants with severe CHD and resemble lesions in pre-term infants. Neuropediatrics. 2006;37(Suppl. 1):S1-183.

12. Miller SP, McQuillen PS, Hamrick S, Xu D, Glidden DV, Charlton N, et al. Abnormal brain development in newborns with congenital heart disease. $N$ Engl J Med. 2007;357:1928-38.

13. Childs AM, Ramenghi LA, Cornette L, Tanner SF, Arthur RJ, Martinez D, et al. Cerebral maturation in premature infants: quantitative assessment using MR imaging. AJNR Am J Neuroradiol. 2001;22:1577-82.

14. Ramenghi LA, Fumagalli M, Righini A, Bassi L, Groppo M, Parazzini C, et al Magnetic resonance imaging assessment of brain maturation in preterm neonates with punctate white matter lesions. Neuroradiology. 2007;49:161-7.

15. Chen CY, Zimmerman RA, Faro S, Parrish B, Wang Z, Bilaniuk LT, et al. MR of the cerebral operculum: topographic identification and measurement of interopercular distances in healthy infants and children. AJNR Am J Neuroradiol. 1995;16:1677-87.

16. Wernovsky G, Shillingford AJ, Gaynor JW. Central nervous system outcomes in children with complex congenital heart disease. Curr Opin Cardiol. 2005;20: 94-9.

17. Hovels-Gurich HH, Konrad K, Skorzenski D, Herpertz-Dahlmann B, Messmer BJ, Seghaye MC. Attentional dysfunction in children after corrective cardiac surgery in infancy. Ann Thorac Surg. 2007;83:1425-30.

18. Shillingford AJ, Glanzman MM, Ittenbach RF, Clancy RR, Gaynor JW, Wernovsky G. Inattention, hyperactivity, and school performance in a population of school-age children with complex congenital heart disease. Pediatrics. 2008; 121:e759-67.

19. Newburger JW, Jonas RA, Soul J, Kussman BD, Bellinger DC, Laussen PC, et al. Randomized trial of hematocrit $25 \%$ versus $35 \%$ during hypothermic cardiopulmonary bypass in infant heart surgery. $J$ Thorac Cardiovasc Surg. 2008;135: $347-54,54$ e $1-4$

20. Wypij D, Jonas RA, Bellinger DC, Del Nido PJ, Mayer JE Jr, Bacha EA, et al. The effect of hematocrit during hypothermic cardiopulmonary bypass in infant heart surgery: results from the combined Boston hematocrit trials. J Thorac Cardiovasc Surg. 2008;135:355-60.

21. Karl TR, Hall S, Ford G, Kelly EA, Brizard CP, Mee RB, et al. Arterial switch with full-flow cardiopulmonary bypass and limited circulatory arrest: neurodevelopmental outcome. J Thorac Cardiovasc Surg. 2004;127:213-22.

22. Freed DH, Robertson CM, Sauve RS, Joffe AR, Rebeyka IM, Ross DB, et al. Intermediate-term outcomes of the arterial switch operation for transposition of great arteries in neonates: alive but well? J Thorac Cardiovasc Surg. 2006;132:845-52.

23. Volpe J. Neurology of the Newborn. 4th ed. Philadelphia: WB Saunders Co; 2001.

24. Song Z, Awate SP, Licht DJ, Gee JC. Clinical neonatal brain MRI segmentation using adaptive nonparametric data models and intensity-based Markov priors. Med Image Comput Comput Assist Interv Int Conf Med Image Comput Comput Assist Interv. 2007;10:883-90.

25. Galli KK, Zimmerman RA, Jarvik GP, Wernovsky G, Kuypers MK, Clancy RR, et al. Periventricular leukomalacia is common after neonatal cardiac surgery. J Thorac Cardiovasc Surg. 2004;127:692-704. 
26. Donofrio MT, Bremer YA, Schieken RM, Gennings C, Morton LD, Eidem BW, et al. Autoregulation of cerebral blood flow in fetuses with congenital heart disease: the brain sparing effect. Pediatr Cardiol. 2003;24: 436-43.

27. Kaltman JR, Di H, Tian Z, Rychik J. Impact of congenital heart disease on cerebrovascular blood flow dynamics in the fetus. Ultrasound Obstet Gynecol. 2005; 25:32-6.

28. Shillingford AJ, Ittenbach RF, Marino BS, Rychik J, Clancy RR, Spray TL, et al. Aortic morphometry and microcephaly in hypoplastic left heart syndrome. Cardiol Young. 2007; 17:189-95.

29. Back SA, Craig A, Luo NL, Ren J, Akundi RS, Ribeiro I, et al. Protective effects of caffeine on chronic hypoxia-induced perinatal white matter injury. Ann Neurol. 2006;60:696-705.

30. Bartlett JM, Wypij D, Bellinger DC, Rappaport LA, Heffner LJ, Jonas RA, et al. Effect of prenatal diagnosis on outcomes in D-transposition of the great arteries. Pediatrics. 2004;113:e335-40.

\section{Discussion}

Dr C. Fraser (Houston, Tex). Dr Licht and colleagues from Children's Hospital of Philadelphia have conducted an outstanding investigation, adding another puzzle piece to understanding the complex interplay between normal brain development and serious congenital heart disease. Term babies with TGA and HLHS have smaller head circumferences and immature brains when compared with their age-matched peers with normal hearts. The maturation scores appear comparable to those of babies with normal hearts at 35 weeks' gestation. Babies with HLHS and TGA have brains that on MRI appear similar to late preterm babies who are otherwise normal. This finding is significant.

Late preterm babies have rapidly maturing brains with white matter predominated by premyelinizing oligodendrocytes. These cells are known to be particularly susceptible to ischemia-reperfusion injury. This must at least, in part, explain the observation of a high incidence of PVL in the study group. These patients' brains may be more susceptible to injury. In preterm and near preterm baby neurologic research, there is a working hypothesis that there is a hierarchy of vulnerability of the maturing brain related to the level of immaturity of the cerebral white matter. The present study suggests that this hierarchy has shifted in the wrong direction by the association of critical congenital heart disease.

To me, one of the most disturbing issues is that despite significant effort by many investigators to modulate the potentially deleterious effects of intraoperative care, particularly limiting the use of hypothermic circulatory arrest, modulating hematocrit level, $\mathrm{pH}$ strategy, and even in the use of selective cerebral perfusion, there are to date no conclusive data that the neurodevelopmental prospects for babies with serious congenital heart disease have improved. There is clearly much work to be done, and I thank Dr Licht and coinvestigators for pushing the field forward.

First, a mechanistic question. The study patients were compared with age-matched controls from historically published data last updated in 2001. Presumably, these control subjects were studied with different MRI methodologies at a different institution. Can we be confident that we are comparing apples to apples given the significant advances that have occurred in MRI technology over the last decade?

Dr Licht. Around this same time period in 2001, Terry Ender was dedicating a lot of her time to structural imaging of prenatal and early preterm babies, and Dr Childs was also doing her work in Leeds in
England. The differences between a 1.5 tesla magnet and a 3 tesla magnet are many, and there are some advantages to 3 tesla and some disadvantages. I think that, for the most part, the TMS looks at structural regions, and so the weighting of the score is weighted for the appearance of the brain and not necessarily something that would be affected by MRI. With 3 tesla we improve the speed of imaging and the contrast to noise, but we may not improve the tissue contrast. There are some advantages at $1.5 \mathrm{~T}$, especially in the neonatal brain, where there is increased water content of the brain, the contrast is improved with volume averaging. Thus, the 3 -mm slices may be actually better or easier to visualize than $1-\mathrm{mm}$ slices.

Overall, I think there are differences in the technique, but I am fairly confident of the results. We would love to be able to recruit our own normal population; however, we are a dedicated children's hospital, and if there is one disadvantage, it is that there are no normal deliveries at our hospital.

Dr Fraser. The second question you have already partially answered, but again, given what you know about these patients now and their vulnerability, let's talk about a hypothetical patient with a prenatally diagnosed transposition. Would you recommend to the attending obstetrician that the child be carried to 40 weeks' gestation or even post-term before elective induction or Cesarean section, rather than 38 weeks, as is often the case?

Dr Licht. I think with the data we have I would make the recommendation to go to as close to term as possible. I think there are some considerations, especially for pregnancies that were diagnosed in remote areas and then referred to a tertiary care hospital. To have a family displaced from their home waiting for a natural labor to be near the hospital, there may be financial and emotional hardships being separated. I also don't think that natural labor at 38 weeks should be tocolyzed to then deliver at 40 weeks. I think it is important to let pregnancies advance with natural labor.

Dr Fraser. Given what you documented in this study and the significant previous contributions on neonatal brain injury from your institution and others, is it justifiable to suggest that the ideal practice for newborns with complex congenital heart disease requiring surgery should include preoperative neurologic imaging, perioperative neurologic monitoring, and long-term neurodevelopmental assessment?

Dr Licht. I am not sure at this time that I would recommend preoperative MRI as a clinically indicated study. The major reasons are that it is very expensive, technically challenging, and involves some risk to the infant; transporting these sick infants out of the cardiac intensive care unit does involve some risk. In the 104 infants who I have imaged preoperatively, 3 infants have had direct benefit from the preoperative imaging, 2 of whom had absent common carotid arteries but continued on to surgery after I informed the surgeon of this anatomic difference. The third patient had an intraparenchymal hemorrhage with layering of blood products that looked very acute, and in this patient we delayed surgery 1 week and then reimaged to make sure that the hematoma had solidified. Therefore, the chance of clinical preoperative imaging directly affecting the outcome is unlikely. However, I believe that preoperative imaging does need to be performed for all neuroprotective studies to get a better sense of the substrate before surgery.

In terms of perioperative monitoring, I think these have a great deal of value, but I don't think they have been studied in the manner that they need to be. They need to be used in a blinded fashion such 
that no clinical decisions are being made on the results of the measurements in such a way that we can better understand what the measurements mean. Near infrared spectroscopy does give you the oxygen saturations but doesn't tell you about the metabolic demand. You can make inferences of cerebral blood flow, but I think we need to know what the measurements mean in a more informed way.

Finally, you asked about neurodevelopment. Long-term neurodevelopment is actually a critical part of all future research. I showed a slide with the Bayley Scales of Infant Development and how these haven't changed much over the course of 20 years, but the Bayley Scales of Infant Development are not a robust test for long-term cognitive outcome, and we really need to be testing the kids at 4 to 5 years to get a better sense of school readiness and to make plans for individualized education programs. I think there should also be multidisciplinary clinics to include endocrinology and psychology, as well as neurology, for the long-term follow-up of these children. 